\title{
Conservational and Sequential Charm Hadronization in Heavy Ion Collisions
}

\author{
Jiaxing Zhao ${ }^{1, *}$, Shuzhe $\mathrm{Shi}^{2}, \mathrm{Nu} \mathrm{Xu}^{3}$, and Pengfei Zhuang ${ }^{1}$ \\ ${ }^{1}$ Physics Department, Tsinghua University and Collaborative Innovation Center of Quantum Matter, \\ Beijing 100084, China \\ ${ }^{2}$ Department of Physics, McGill University, Montréal, QC H3A 2T8, Canada \\ ${ }^{3}$ Institute of Modern Physics, Chinese Academy of Sciences, Lanzhou, Gansu 730000, China
}

\begin{abstract}
Heavy quarks are almost initially produced and the number is conserved during the evolution of the hot medium produced in Heavy Ion collisions. We firstly take into account the charm conservation effect on charm hadron hadronization via sequential coalescence model. In the frame of sequential coalescence, the charm conservation enhances the earlier hadron production and suppresses the later production. This relative enhancement (suppression) changes significantly the charmed hadron ratios in heavy ion collisions at RHIC and LHC.
\end{abstract}

Because the charm quark mass $m_{c} \sim 1.2 \mathrm{GeV}$ is much larger than the typical temperature of the fireball formed in heavy ion collisions at RHIC and LHC energies, the charm quark number is almost contributed by the initial production and conserved during the whole evolution of the colliding system [1]. The precise measurement of $D_{s}, D^{0}$ and charm baryons in experiment give us a chance to study the charm conservation and charm hadronization mechanism in Heavy Ion Collisions. Recently, the yield ratio of $D_{s}^{+} / D^{0}$ is measured in heavy ion collisions at RHIC [2] and LHC [3] energies. However, the theoretical results seems underestimate the experiment data in the applicable kinematic region [4]. We emphasis that we need consider the effect of charm conservation in charmed hadron production [5-7]. Qualitatively, when the charm conservation is taken into account, the strangeness enhancement induced $D_{s}^{+}$ enhancement results in a $D^{0}$ suppression, which will lead to a further $D_{s}^{+} / D^{0}$ enhancement!

In our previous study [8], we take into account the charm conservation effect via sequential coalescence model. Its first time to consider charm conservation self-consistently in charm hadron production in Heavy Ion Collisions. And I will introduce the framework of the model and results in this paper.

By solving the two-body Dirac equation, which was successfully applied to the relativistic description of light meson spectra [9] in vacuum and extended to hidden and open[10] charmed mesons at finite temperature, we obtain the charmed meson binding energy $\epsilon_{m}(T)$ and the radial wave function $\psi(r, T)$. From the definition of meson melting $\epsilon_{m}\left(T_{m}\right)=0$ or $\left\langle r_{m}\right\rangle\left(T_{m}\right) \rightarrow \infty$, we extract the meson dissociation temperatures $T_{D_{s}^{+}}=1.2 T_{c}$ and $T_{D_{s}^{*+}} \simeq T_{D^{0}} \simeq T_{D^{* 0}} \simeq T_{D^{*+}}=1.15 T_{c}$, as showed in Fig.1. Considering the relation $V_{q q} \simeq V_{q \bar{q}} / 2$ between attractive quark-quark and quark-anti-quark potentials, the dissociation temperature of charmed baryons like $\Lambda_{c}, \Sigma_{c}, \Xi_{c}$ and $\Omega_{c}$ should be lower than that for

\footnotetext{
*e-mail: zhao-jx15@mails.tsinghua.edu.cn
} 
charmed mesons [11]. Because the effect of charm conservation on hadron production depends mainly on the order of productions, we take simply the coalescence temperature for charmed baryons as $T_{c}$ in the following calculation.
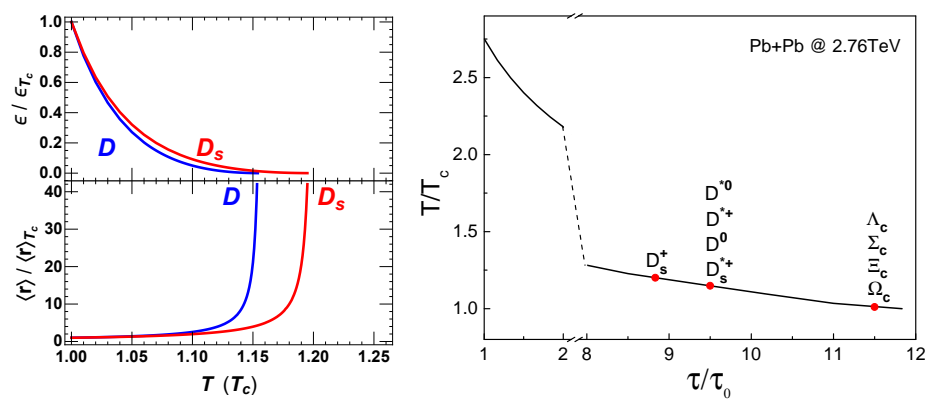

Figure 1. (Left) The binding energy and average radius of charm meson $D^{0}$ and $D_{s}$. (Right)The time evolution of the maximum temperature in central $\mathrm{Pb}+\mathrm{Pb}$ collisions at $\sqrt{s_{N N}}=2.76 \mathrm{TeV}$. The dots indicate the coalescence temperature (time) for charmed hadrons. $\tau_{0}=0.6 \mathrm{fm} / \mathrm{c}$ and $T_{c}=165 \mathrm{MeV}$ are the initial time and critical temperature of the quark matter.

The evolution of quark matter created in high energy nuclear collisions can described by the hydrodynamic equations $\partial_{\mu} T^{\mu v}=0[12,13]$. During the evolution of the quark matter, the temperature continuously drops down due to the expansion of the system. When the local temperature $T\left(\mathbf{x}, \tau_{h}\right)$ reaches the value of the dissociation temperature $T_{h}$ for a kind of charmed hadrons, the corresponding hadrons will be produced. Aim to see clearly the hadronziation sequential of charmed hadrons, we showed the time evolution of the temperature at the center of the fireball in central $\mathrm{Pb}+\mathrm{Pb}$ collisions at LHC energy and the hadronization times $\tau_{h}$ of $D_{s}^{+}, D^{0}\left(D_{s}^{*+}, D^{* 0}, D^{*+}\right)$ and charmed baryon $\Lambda_{c}\left(\Sigma_{c}, \Xi_{c}, \Omega_{c}\right)$ in right hand side of Fig.1.

The coalescence mechanism is successfully used to describe hadron distributions in heavy ion collisions, like the quark number scaling law of hadron collective flow $[14,15]$, the enhancement of baryon to meson ratios [16-18], the yield. We built the sequential coalescence model with charm conservation to calculate the charmed hadron spectra.

$$
\frac{d N_{h}}{d^{2} P_{T} d \eta}=C \int P^{\mu} d \sigma_{\mu} \prod_{i=1}^{n} \frac{d^{4} x_{i} d^{4} p_{i}}{(2 \pi)^{3}} f_{i}\left(x_{i}, p_{i}\right) \times W_{h}\left(x_{1}, \ldots, x_{i}, p_{1}, \ldots, p_{i}\right),
$$

where the integration is on the coalescence hypersurface $\sigma_{\mu}\left(\tau_{h}, \mathbf{X}\right)$, the summation is over the constituent quarks with $n=2$ for mesons and $n=3$ for baryons, and $P_{\mu}=\left(P_{0}, \mathbf{P}\right)$ is the four dimensional hadron momentum. The constant $C$ is the statistical factor to take into account the internal quantum numbers in forming a colorless hadron. Where $W_{h}$ is the Wigner function or coalescence probability for $n$ quarks to combine into a hadron and the quark distributions. It can be constructed directly from the wavefunction solved by the twobody Dirac equation $[19,20] . f_{i}$ in the spectra (1) is the distribution function of the constituent quarks in phase space. The light quarks $u$ and $d$ are controlled by the Fermi-Dirac distribution $f_{q}(x, p)=N_{q} /\left(e^{u_{\mu} p^{\mu} / T}+1\right)$. Considering that strange quarks may not reach fully chemical equilibrium at RHIC energy, the fugacity factor $\gamma_{s}$ is included and $\gamma_{s}=1 \mathrm{in} \mathrm{LHC} \mathrm{energies.}$

The experimentally measured $D$ meson collective properties at $p_{T}<2 \mathrm{GeV} / \mathrm{c}$ [21], suggests the slowly moving charm quarks may reach thermalization with mdium. Therefore, we 

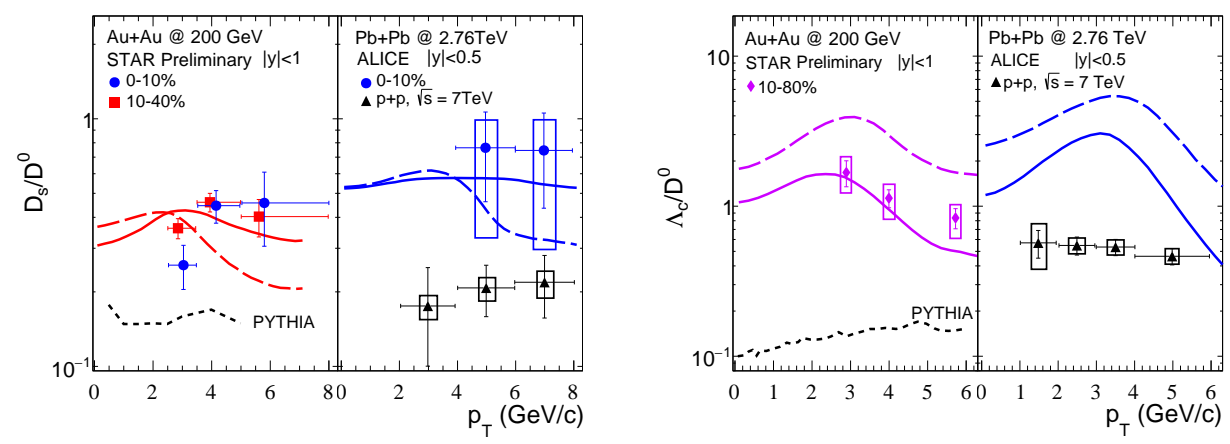

Figure 2. (Left)The yield ratio of $D_{s}^{+} / D^{0}$ at RHIC and LHC energies. The experimental data in A+A and $p+p$ collisions are from STAR [2] and ALICE $[3,26]$ Collaborations, the dotted line is the PYTHIA simulation for $\mathrm{p}+\mathrm{p}$ collisions [2], and the solid and dashed lines are respectively our sequential and simultaneous coalescence calculations. (Right)The yield ratio of $\Lambda_{c}^{+} / D^{0}$ at RHIC and LHC energies. The experimental data in A+A and $p+p$ collisions are from STAR [27, 28] and ALICE [29] collaborations, the dotted line is the PYTHIA simulation for $p+p$ collisions [27], and the solid and dashed lines are respectively our sequential and simultaneous coalescence calculations.

take, as a first approximation, a linear combination of $f_{p p}$ and $f_{t h}$ as the charm quark distribution, $f_{c}(x, p)=\rho_{c}(x)\left[\alpha f_{t h}(p)+\beta f_{p p}(p)\right]$. The coefficients $\alpha$ and $\beta$ control the degree of thermalization of charm quarks, reflect the continuous thermalization charm quarks in hot medium. We have evolute charm quark in hot medium with Langevin equation in further study. We treat the charm quark density in coordinate space $\rho_{c}(x)$ as a superposition of $\mathrm{p}+\mathrm{p}$ collisions [22],

$$
\rho_{c}(x \mid \mathbf{b})=r(\tau) T_{A}\left(\mathbf{x}_{T}\right) T_{B}\left(\mathbf{x}_{T}-\mathbf{b}\right) \frac{\cosh \eta}{\tau} \frac{d \sigma_{p p}^{c \bar{c}}}{d \eta},
$$

where $T_{A}$ and $T_{B}$ are thickness functions of the two colliding nuclei [23], $\mathbf{b}$ is the impact parameter of the collision, $d \sigma_{p p}^{c \bar{c}} / d \eta$ is the rapidity distribution of charm quarks in $\mathrm{p}+\mathrm{p}$ collisions, and the factor $1 / \tau$ comes from the Bjorken expansion mechanism [24]. The time dependent charm quark number fraction $r(\tau)$ describes the charm conservation during the sequential coalescence,

$$
r(\tau)= \begin{cases}1 & \tau \leq \tau_{D_{s}^{+}} \\ 1-N_{D_{s}^{+}} / N_{c} & \tau_{D_{s}^{+}}<\tau \leq \tau_{D^{0}} \\ 1-N_{D} / N_{c} & \tau_{D^{0}}<\tau\end{cases}
$$

where $N_{D}=N_{D_{s}^{+}}+N_{D^{0}}+N_{D_{s}^{*+}}+N_{D^{* 0}}+N_{D^{*+}}$ is the total $D$ meson number or charm quark number. If all the charmed hadrons are simultaneously produced at the phase boundary (a unified coalescence temperature $T_{c}$ or coalescence time $\tau_{c}$ in usual coalescence models), the charm fraction $r(\tau)=1$ becomes time independent during the evolution of the quark matter.

Firstly, aim to see clearly the effect of sequential coalescence with charm conservation, we calculated the yield ratio of $D_{s}^{+} / D^{0}$, as showed in Fig.2. In comparison with the $\mathrm{p}+\mathrm{p}$ collisions (PYTHIA simulation at RHIC and data at LHC), the ratio in A+A collisions is strongly enhanced. The first reason for this large enhancement is the $D_{s}$ enhancement due to the thermal strange quark production in quark matter [25]. The ratio at RHIC can increase 
from PYTHIA value $\sim 0.15$ to $0.2-0.3$ in a coalescence model [4]. In our model, both the strangeness enhancement and the charm conservation are responsible for the ratio enhancement. On one hand, the earlier $D_{s}^{+}$production is with $100 \%$ of charm quarks and the later $D^{0}$ production is with only $\sim 90 \%$ of charm quarks. Therefore, the $D_{s}^{+}$enhancement leads to a $D^{0}$ suppression and in turn to a further $D_{s}^{+} / D^{0}$ enhancement. On the other hand, the later $D^{0}$ production is with more thermalized(softer) charm quarks, its distribution is then shifted to the lower $p_{T}$ region. As a competition of these two effects, the ratio $D_{s}^{+} / D^{0}$ will be significantly enhanced at high $p_{T}$ and may weakly suppressed at low $p_{T}$. Here we calculated the ratio in two cases: One (solid lines) is in the frame of sequential coalescence and the other (dashed lines) is with a simultaneous coalescence at the phase boundary. It's clear that, the sequential coalescence with charm conservation induces a strong enhancement at high $p_{T}$ and a slight suppression at low $p_{T}$ and this can explain experiment date quite well.

Then, we turn to study the charmed baryon production. Considering the difference in statistics for two- and three-body states shown in the hadron spectra (1), the baryon to meson ratio in $\mathrm{A}+\mathrm{A}$ collisions will dramatically enhanced in comparison with $\mathrm{p}+\mathrm{p}$ collisions in coalescence models. But, in our model, the big difference between the charm quark number fraction $r=0.6$ for $\Lambda_{c}$ production and $r=0.9$ for $D^{0}$ production strongly weakens the enhancement of the ratio at any $p_{T}$, see the solid lines in comparison with the dashed lines. As showed in Fig.2, our prediction about the yield ratio of $\Lambda_{c}^{+} / D^{0}$ is agree well with preliminary experiment data from STAR.

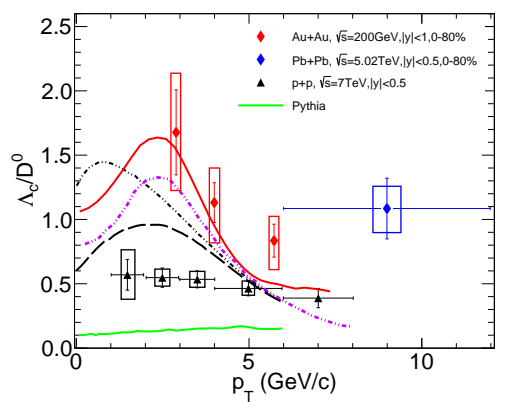

Figure 3. The yield ratio of $\Lambda_{c}^{+} / D^{0}$ at RHIC (Red dimond) and LHC (Blue dimond) energies. The black triangle represent the ratio of $\Lambda_{c}^{+} / D^{0}$ in $p p$ collisions at $7 \mathrm{TeV}$. The green line is the PYTHIA simulation for $p p$ collisions at $200 \mathrm{GeV}$, and the dotted and dashed line are respectively Greco's and Ko's results. The red solid line is the result from our model.

Finally, we would like to draw an attention to the behavior of $\Lambda_{c}^{+} / D^{0}$ ratio at high $p_{T}$. We combine both AA data and pp data from RHIC and LHC, showed in Fig.3. The theoretical results of W. Greco [7], Ko [6] and ours underestimate the experimental data at $p_{T}$ larger than $6 \mathrm{GeV}$ obviously. And PYTHIA simulation seems fail to reproduce the experiment data of $p+p$ collisions at 7TeV. That indicate the mechanism of charm fragment to $\Lambda_{c}$ need to modified. Adding the correct contribution of fragmentation at high $p_{T}$, we believe the experiment data will be explained well in Heavy Ion collisions.

In summary, we established a sequential hadronization model with charm conservation and applied it into charm hadron production in Heavy Ion Collisions. It is a self-consistently way to describe charm hadron production. The charm conservation leads to an enhancement for earlier produced hadrons and a relative suppression for later produced hadrons. And our 
results on $D_{s} / D^{0}$ and $\Lambda_{c} / D^{0}$ ratio consistent with experiment data. The same frame can also be used to describe the bottom hadrons production in Heavy Ion Collisions.

\section{References}

[1] K.Zhou, Z.Chen, C.Greiner and P.Zhuang, Phys. Lett. B758, 434(2016).

[2] L. Zhou [STAR Collaboration], Nucl. Phys. A 967, 620 (2017).

[3] J. Adam et al. [ALICE Collaboration], JHEP 1603, 082 (2016).

[4] M.He, R.Fries and R.Rapp, Phys. Rev. Lett. 110, 112301(2013).

[5] M.Gorenstein, A.Kostyuk, H.Stoecker and W.Greiner, Phys. Lett. B509, 277(2001).

[6] Y.Oh, C.Ko, S.Lee and S.Yasui, Phys. Rev. C79, 044905(2009).

[7] S. Plumari, V. Minissale, S. K. Das, G. Coci and V. Greco, Eur. Phys. J. C 78, no. 4, 348 (2018).

[8] J. Zhao, S. Shi, N. Xu and P. Zhuang, arXiv:1805.10858 [hep-ph].

[9] H. W. Crater, J. H. Yoon and C. Y. Wong, Phys. Rev. D 79, 034011 (2009); H. Crater and P. Van Alstine, Phys. Rev. D 70, 034026 (2004).

[10] S. Shi, X. Guo and P. Zhuang, Phys. Rev. D 88, no. 1, 014021 (2013); X. Guo, S. Shi and P. Zhuang, Phys. Lett. B 718, 143 (2012).

[11] J.Zhao and P.Zhuang, Few Body Syst. 58, 100(2017).

[12] H.Song, S.Bass, U.Heinz, T.Hirano and C.Shen, Phys. Rev. Lett. 106, 192301(2011).

[13] T.Hirano, P.Huovinen and Y.Nara, Phys. Rev. C83, 021902(2011).

[14] D.Molnar and S.Voloshin, Phys. Rev. Lett. 91, 092301(2003).

[15] V.Greco, C.Ko and P.Levai, Phys. Rev. C68, 034904(2003).

[16] R.Hwa and C.Yang, Phys. Rev. C67, 034902(2003).

[17] R.Fries, B.Muller, C.Nonaka and S.Bass, Phys. Rev. Lett. 90, 202303(2003).

[18] V.Greco, C.Ko and P.Levai, Phys. Rev. Lett. 90, 202302(2003).

[19] J. Zhao and B. Chen, Phys. Lett. B 776, 17 (2018).

[20] J. Zhao, H. He and P. Zhuang, Phys. Lett. B 771, 349 (2017).

[21] L.Adamczyk et al. [STAR Collaboration], Phys. Rev. Lett. 118, 212301(2017).

[22] K. Zhou, N. Xu, Z. Xu and P. Zhuang, Phys. Rev. C 89, no. 5, 054911 (2014).

[23] M. L. Miller, K. Reygers, S. J. Sanders and P. Steinberg, Ann. Rev. Nucl. Part. Sci. 57, 205 (2007).

[24] J. D. Bjorken, Phys. Rev. D 27, 140 (1983).

[25] G. Agakishiev et al. [STAR Collaboration], Phys. Rev. Lett. 108 (2012) 072301.

[26] B. Abelev et al. [ALICE Collaboration], Phys. Lett. B 718, 279 (2012).

[27] G. Xie [STAR Collaboration], Nucl. Phys. A 967, 928 (2017).

[28] Z. Ye. 2018. [STAR Collaboration], talk at Quark Matter, Italy.

[29] S. Acharya et al. [ALICE Collaboration], arXiv:1712.09581 [nucl-ex]. 